\title{
Genetic Algorithm for Thinning Gray-Scale Images of Characters
}

\author{
Tadayoshi Shioyama, Akira Okumura and Yoshikazu Aoki \\ Department of Mechanical and System Engineering \\ Kyoto Institute of Technology, Sakyo - ku, Kyoto 606
}

\begin{abstract}
In this paper, we propose a new method for thinning digital gray-scale pictures by fitting piece-wise linear line to skelton. The method is not based on the point-wise gray-value information as Salari and Siy method, but based on an objective function robust for noise contaminated pictures. To optimize the function, we search the solution of the optimization problem with genetic algorithm. The procedure of the method is as follows. We allocate small regions so that they cover the neighborhood of skelton of object. In each small region, we extract a linear line which approximates the skelton in the small region, and represent the line by a couple of label numbers of points with nonzero gray values through which the line passes. A string is constructed by collecting all the label pairs. The fitness of a string is given by an objective function which is constructed so as to evaluate the proximity to skelton and to be robust for noise. After a genetic algorithm generates successive populations of strings, a skelton is given by the string with the greatest fitness.
\end{abstract}

\section{Introduction}

The transformation of images of linelike objects such as printed or handprinted characters and chromosomes to their skeltons is a problem of great importance in pattern recognition. In pattern recognition, the thickness of the lines which constitute the objects, is not effective, but the skeltons are used to extract topological features of the objects.

In methods for thinning objects in black and white (binary) pictures, the skelton is obtained from the boundary of the binary picture. However, the boundary is not well defined for many applications such as handwritten characters and chromosomes. Salari and Siy [1] have proposed a method for thinning digital gray-scale pictures. In their method, the skelton is obtained by seeking the ridge (high gray value) areas to position the skelton. In their thinning process, the boundary point of object area which is a set of connected elements with nonzero gray values, is removed using the gray value information of its eight neighbors if the conditions for retaining structure information and 
topological properties are satisfied. Hence their method which is based on the point-wise gray value information, is considered to have a drawback that it is not robust for noise contaminated images of objects.

In the present paper, we propose a new method for thinning digital gray-scale pictures by fitting piece-wise linear line to the ridge i.e. skelton. The method is not based on the point-wise gray value information but based on the noise resistive objective function. To optimize the function, we search the solution of the optimization problem by genetic algorithm [2] which surpasses traditional search procedures in the quest for robustness due to parallel flavor. The procedure of the present method is as follows. We allocate small regions so that they cover the neighborhood of skelton of object. In each small region, we extract a linear line which approximates the skelton in the small region, and represent the line by a couple of label numbers of points with nonzero gray values determining the line. A string is constructed by collecting all the label pairs. The fitness of a string is given by an objective function which is constructed so as to evaluate the proximity to skelton and to be robust for noise. After a genetic algorithm composed of three operators: reproduction, crossover and mutation, generates successive populations of strings, a skelton is obtained from the string with the greatest fitness. To evaluate the present method, the resulting skeltons are shown for noise contaminated original pictures.

\section{The Piece-Wise Linear Line Fitting Method}

\subsection{Elementary Objective Function}

Let $f(x, y)$ be the gray value at a point with image coordinate $(x, y)$. Denote by $m_{p q}$ the moment of order $\mathrm{p}+\mathrm{q}$ of a gray value function $\mathrm{f}(\mathrm{x}, \mathrm{y})$ around a point $\left(x_{0}, y_{0}\right): m_{p q}=\int\left(x-x_{0}\right)^{p}\left(y-y_{0}\right)^{q} f(x, y) d x d y, \quad p+q \geq 0$, by $\tilde{m}_{p q}$ the normalized moment: $\tilde{m}_{p q}=m_{p q} / m_{00}$. Define an elementary objective function $\phi$ as $\phi=\sqrt{\tilde{m}_{10}^{2}+\tilde{m}_{01}^{2}}$. The function $\phi$ takes a small value at the ridge (high gray value) areas. Then, the function $\phi$ can be used to evaluate the proximity of skelton. The function $\phi$ is considered to be robust for noise contaminated images because it is based on moments.

In practical computation, the function $\phi$ is obtained by computing the moments in the $5 \times 5$ mask with the center at the considered point $\left(x_{0}, y_{0}\right)$. Figure 1 shows the perspective of the value of $\phi$ for character " $A$ ", where for convenience, $\phi$ is set as zero at $\left(x_{0}, y_{0}\right)$ such that $f\left(x_{0}, y_{0}\right)=0$. 


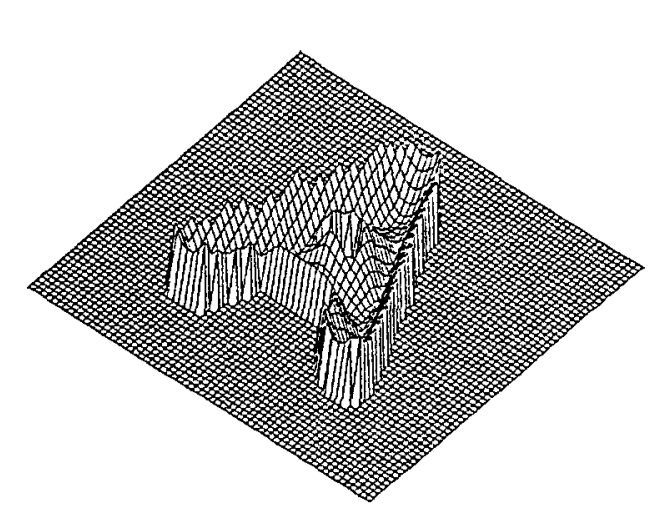

Fig.1 The perspective of $\phi$ for character "A".

The function $\phi$ is used for two purposes. First, it is used to determine whether the considered point $\left(x_{0}, y_{0}\right)$ is in the neighborhood of skelton or not. That is, the point $\left(x_{0}, y_{0}\right)$ is decided to be in the neighborhood of skelton if the function $\phi$ is less than a threshold $\theta$. The threshold $\theta$ is determined by $\mathrm{P}$-tile method in which the area of histogram of values of $\phi$ less $\operatorname{than} \theta$ is t-percent of the area of total histogram of values of $\phi$. In the sequel, $t$ is set to be 50 percent. Then, $\theta$ is determined to be 1.06 from values of $\phi$ for 26 alphabetical capital letters. Second, the function $\phi$ is used to construct an evaluation(objective function) of string as described in the later section.

\subsection{Allocating Small Regions}

Small regions of size $w \times w$ are allocated by raster scanning without overlapping each other so that each small region contains more points than a threshold, which are in the neighborhood of skelton.

In each small region, a linear line is extracted by choosing two points with nozero gray values in the small region such that the extracted line passing through the two points is the best approximation to skelton in the small region.

\subsection{Thinning with GA}

\subsubsection{String}

Let $\mathrm{N}$ denote the number of allocated small regions, and $k_{i}, \mathrm{i}=1, \ldots, \mathrm{N}$, the number of points with nonzero gray values in the $\mathrm{i}$-th small region, where each point is labelled by a number in a set $\left\{1,2, \ldots, k_{i}\right\}$. Denote by $L_{i}^{1}$ and $L_{i}^{2}$, labels of two points which specify a linear line in the $\mathrm{i}$-th small region. A string is given by collecting the label couple $\left(L_{i}^{1}, L_{i}^{2}\right), \mathrm{i}=1, \ldots, \mathrm{N}$, i.e. $\left(L_{1}^{1} L_{1}^{2} L_{2}^{1} L_{2}^{2} \ldots L_{i}^{1} L_{i}^{2} \ldots L_{N}^{1} L_{N}^{2}\right)$.

\subsubsection{Evaluation of String}

Let a linear line $P_{i}$, which is extracted in the $\mathrm{i}$-th small region, be composed of a set of $n_{i}$ points as follows: $P_{i}=\left\{p_{i}^{1}, p_{i}^{2}, \ldots, p_{i}^{n_{i}}\right\}, i=1, \ldots, N$, where the points $p_{i}^{1}$ and $p_{i}^{n_{i}}$ are on the boundary of $i$-th small region. Define the objective function 
$E\left(P_{i}\right)$ of a linear line $P_{i}$ as

$$
E\left(P_{i}\right) \equiv \frac{1}{n_{i}} \sum_{j=1}^{n_{i}} \phi\left(p_{i}^{j}\right) .
$$

The objective function $E_{\phi}$ of a string is defined as $E_{\phi} \equiv \frac{1}{N} \sum_{i=1}^{N} E\left(P_{i}\right)$. To evaluate the connectivity of the thinning results, we define the objective function $E_{c}$ as follows. Assume that the $\mathrm{j}$-th small region is adjacent to the considered i-th small region. Let $P_{i j}$ denote the linear line passing through two centers of the $\mathrm{i}$-th and $\mathrm{j}$-th small regions. Define $\theta_{i j}$ as an angle between $P_{i}$ and $P_{j}$, and $\rho_{i j}$ as an angle between $P_{i}$ and $P_{i j}$. Define $x_{i j}$ as

$$
\begin{gathered}
x_{i j}=0 \text { for } 0 \leq \min \left(\rho_{i j}, \pi-\rho_{i j}\right)<\pi / 4 \\
x_{i j}=1 \text { for } \pi / 4 \leq \min \left(\rho_{i j}, \pi-\rho_{i j}\right) \leq \pi / 2 .
\end{gathered}
$$

Then, we define the evaluation $V_{i j}$ for connectivity between the $\mathrm{i}$-th and $\mathrm{j}$-th small regions as $V_{i j} \equiv x_{i j}\left(1+\cos \theta_{i j}\right)+\left(1-x_{i j}\right)\left(1-\cos \theta_{i j}\right)$. We define the objective function $E_{c}$ for connectivity of string as $E_{c} \equiv \frac{1}{N} \sum_{i=1}^{N} \sum_{j=1}^{m_{i}} V_{i j}$, where $m_{i}$ denotes the number of small regions adjacent to the $\mathrm{i}$-th small region. Hence, the objective function $\mathrm{E}$ of a string is defined as $E \equiv E_{\phi}+\alpha E_{c}$, where a constant $\alpha$ is a weighting coefficient. The small $E$ implies the greater fitness of string.

\subsubsection{Reproduction}

Strings with lower fitness are selected and cannot have ability to contribute offspring in the next generation. The ratio of the number of such inferior strings to the population size is called generation gap. The generation gap of strings are supplemented by copying strings with higher fitness to retain the population size. In reproduction process, strings are copied according to their objective function values.

\subsubsection{Crossover and Mutation}

Uniform crossover proceeds on mated string couple among a population. That is, exchange is performed in uniform probability on a label-by-label basis. The subsequent operator, mutation, is performed on a label-by-label basis on the created two new strings.

After a genetic algorithm composed of three operators: reproduction, crossover and mutation, generates successive populations of strings, a thinning result is obtained from the string with the greatest fitness.

\subsection{Postprocessing}

Postprocessing is performed in the following steps to guarantee a connectivity of thinning result. 
(step 1) Find an end point $P$ in the thinning result. Set a $2 w \times 2 w$ mask with center at the end point.

(step 2)In the mask, find end points other than $\mathrm{P}$ which satisfy the following two conditions. If exist, choose an end point $Q$ among them such that the linear line $P Q$ has the least objective function value defined as (1). Then, connect the end points $Q$ and $P$ by a linear line.

Condition 1. The point is not connected with $P$ in the $2 \mathrm{w} \times 2 \mathrm{w}$ mask.

Condition 2. The linear line determined by the point and $\mathrm{P}$, has an objective function value given by (1) less than a threshold.

If there is not such end point other than $P$, go to step 3 .

(step 3) In the image of thinning result, choose the black point $Q$ such that it satisfies the above two conditions and moreover the linear line $P Q$ has the least objective function value given by (1). Connect $\mathrm{P}$ and $\mathrm{Q}$ by a linear line.

These steps are performed for every end point $P$.

\section{Results}

The present thinning method is evaluated using $64 \times 64$ original image with 16 gray levels with additive gaussian noise of mean 0 and variance 1 . In the experiment, the size of small region is set as $6 \times 6$, and the following parameters in GA are used: The population size $=100$, The number of generations $=500$, The probability of crossover $=0.8$, The probability of mutation $=0.003$, and The generation gap $=0.4$. Figure 2 shows original images. Figure 3 shows the results by the present thinning method. For comparison, the results by the Salari and Siy method are shown in Fig.4. Here, the procedure where noisy isolated points are removed, is performed only in Fig.4. In the results by Salari and Siy method, there are improper protrusions. From these results, it is found that the present thinning method is robust even for noise contaminated images.
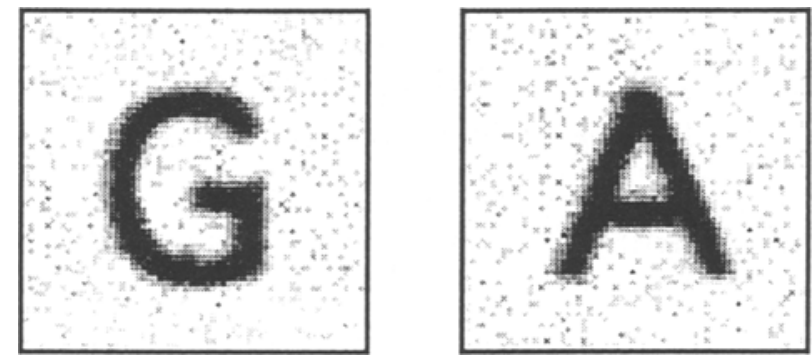

Fig.2 Original images. 

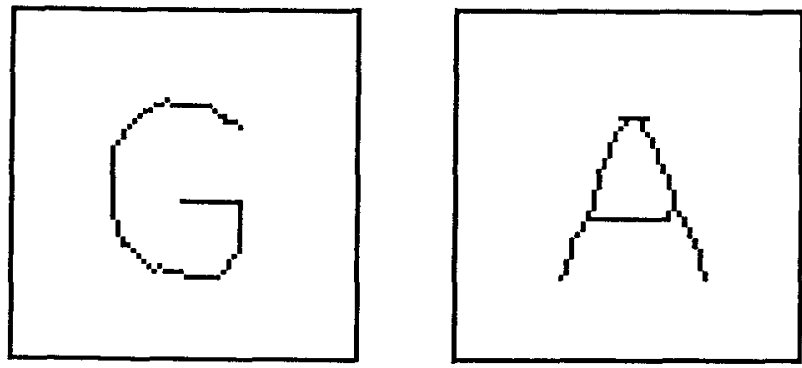

Fig.3 The thinning results by the present method.
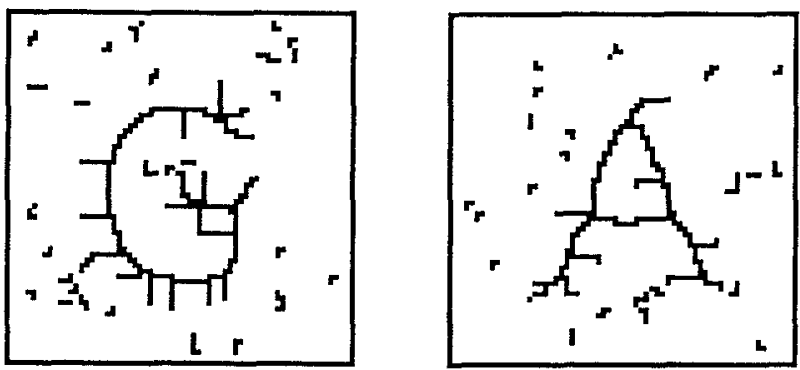

Fig.4 The thinning results by Salari and Siy method.

\section{Conclusion}

We have proposed a new method with GA for thinning digital gray-scale pictures by fitting piece-wise linear line to skelton. The method is not based on point-wise gray value information but based on a noise resistive objective function. To optimize the function, we have searched the solution with GA. In comparison with the Salari and Siy method which is a typical conventional method, the present method is found to be robust even for noise contaminated pictures. The computational burden required by the present algorithm is heavy. It is the problem to overcome the drawback.

\section{References}

[1] E.Salari and P.Siy:The Ridge-Seeking Method for Obtaining the Skelton of Digital Images. IEEE Trans. on Systems, Man, and Cybernetics, SMC-14, 524-528 (1984)

[2] D.E.Goldberg:Genetic Algorithms in Search, Optimization, and Machine Learning. Addison-Wesley 1989 